\title{
Stage IIIA Eyelid Carcinoma AJCC v7
}

National Cancer Institute

\section{Source}

National Cancer Institute. Stage IIIA Eyelid Carcinoma A/CC v7. NCI Thesaurus. Code C88129.

Stage IIIA includes: T3b, N0, MO. T3b: Complete tumor resection requires enucleation, exenteration, or bone resection. N0: No regional lymph node metastasis. M0: No distant metastasis. (AJCC 7th ed.) 